\title{
Extreme Ultraviolet Laser Excites Atomic Giant Resonance
}

\author{
M. Richter, ${ }^{1, *}$ M. Ya. Amusia, ${ }^{2}$ S. V. Bobashev, ${ }^{2}$ T. Feigl, ${ }^{3}$ P. N. Juranić, ${ }^{4}$ M. Martins, ${ }^{5}$ A. A. Sorokin,, 2 and K. Tiedtke ${ }^{4}$ \\ ${ }^{1}$ Physikalisch-Technische Bundesanstalt, Abbestraße 2-12, 10587 Berlin, Germany \\ ${ }^{2}$ Ioffe Physico-Technical Institute, Polytekhnicheskaya 26, 194021 St. Petersburg, Russia \\ ${ }^{3}$ Fraunhofer-Institut für Angewandte Optik und Feinmechanik, Albert-Einstein-Straße 7, 07745 Jena, Germany \\ ${ }^{4}$ Deutsches Elektronen-Synchrotron, Notkestraße 85, 22603 Hamburg, Germany \\ ${ }^{5}$ Universität Hamburg, Institut für Experimentalphysik, Luruper Chaussee 149, 22761 Hamburg, Germany
}

(Received 15 January 2009; published 24 April 2009)

\begin{abstract}
Exceptional behavior of light-matter interaction in the extreme ultraviolet is demonstrated. The photoionization of different rare gases was compared at the free-electron laser in Hamburg, FLASH, by applying ion spectroscopy at the wavelength of $13.7 \mathrm{~nm}$ and irradiance levels of thousands of terawatts per square centimeter. In the case of xenon, the degree of nonlinear photoionization was found to be significantly higher than for neon, argon, and krypton. This target specific behavior cannot be explained by the standard theories developed for optical strong-field phenomena. We suspect that the collective giant $4 d$ resonance of xenon is the driving force behind the effect that arises in this spectral range.
\end{abstract}

The new Free-Electron Laser (FEL) in Hamburg FLASH [1] currently produces the highest irradiance of extreme ultraviolet (EUV) and soft x-ray pulses in the world. $10^{16} \mathrm{~W} \mathrm{~cm}^{-2}$ were recently achieved in a photoionization experiment performed at FLASH on xenon atoms in the EUV wavelength region at about $13 \mathrm{~nm}$ [2]. Under these extreme conditions of ultrahigh intensities at short wavelengths, the mechanisms of light-matter interaction are not well understood, as the above xenon study clearly demonstrates. Here, we show now that the nature of the EUV laser light on the interaction, i.e., as weak perturbation in terms of individual photons or as a strongly interacting electromagnetic wave, depends significantly on the electron structure of the target and the excitation of strong resonances. The quiver energy transferred to free electrons which defines the degree of perturbation of matter by powerful lasers in the optical regime [3-5] plays a minor role in the EUV. This comes out by comparing the nonlinear photoionization of different rare gases by ion mass-to-charge spectroscopy. Our experiments were performed at FLASH at the wavelength of $13.7 \mathrm{~nm}$. The work refers to the fundamental aspects of the photoelectric effect [6] and is significant for any investigation on nanometer and femtosecond scales using $\mathrm{x}$-ray lasers [7-11].

The gases were investigated under equivalent conditions in the microfocus region of a spherical multilayer mirror developed for EUV lithography [12]. The mirror could be moved along the FEL beam in order to shift the focus in back-reflection geometry into and out of the interaction volume of our ion time-of-flight (TOF) spectrometer $[2,13-15]$ and to vary the effective FEL beam diameter from 4 to $200 \mu \mathrm{m}$ for our measurements. As a result, the peak irradiance of the FEL pulses with pulse durations in the order of $10 \mathrm{fs}$ could be varied from $10^{12}$ to $2 \times$ $10^{15} \mathrm{~W} \mathrm{~cm}^{-2}$. The respective target gas filled the experi- mental vacuum chamber homogeneously at the considerably low pressure of about $10^{-4} \mathrm{~Pa}$ to avoid any interaction between neighboring atoms and ions. Absolute FEL pulse energy in the microjoule regime was monitored with a relative standard uncertainty of $15 \%$ on a shot-to-shot basis by means of calibrated gas-monitor detectors $[16,17]$.

Figure 1 shows ion TOF spectra of xenon measured at the wavelength of $13.7 \mathrm{~nm}$, i.e., at the photon energy of $90.5 \mathrm{eV}$, at considerably low irradiance of $(2.5 \pm 0.7) \times$ $10^{12} \mathrm{~W} \mathrm{~cm}^{-2}$ [Fig. 1(a)] and at high irradiances of (1.7 \pm $0.5)$ and $(2.0 \pm 0.6) \times 10^{15} \mathrm{~W} \mathrm{~cm}^{-2}$ [Figs. 1(b) and 1(c)]. The spectra confirm our former results obtained at the wavelength of $13.3 \mathrm{~nm}$, i.e., $93-\mathrm{eV}$ photon energy [2]. At low irradiance, the photoionization of xenon (ground state electron configuration $\mathrm{Xe}$ : $[\mathrm{Kr}] 4 d^{10} 5 s^{2} 5 p^{6}$ ) is dominated by the so-called giant $4 d \rightarrow \varepsilon f$ continuum resonance which ranges from 14 to $11 \mathrm{~nm}$, i.e., from about 88 to $113 \mathrm{eV}$ photon energy [18]. Here, it is a one-photon process and leads via Auger decay mainly to doubly or triply charged final states with two or three electron vacancies in the outer $5 p$ shell [19]. Direct $5 p$ emission ending up in a singly charged final state plays a minor role. At $1.7 \times 10^{15} \mathrm{~W} \mathrm{~cm}^{-2}$ [Fig. 1(b)], on the other hand, charge states up to $\mathrm{Xe}^{14+}$ occur. Here, to achieve this highly charged state, a single $\mathrm{Xe}$ atom must have absorbed 22 EUV photons of $90.5 \mathrm{eV}$ each, or $1.93 \mathrm{keV}$ total [20], during a FEL pulse with a duration of $10 \mathrm{fs}$ [1]. At $2.0 \times$ $10^{15} \mathrm{~W} \mathrm{~cm}^{-2}$, even $\mathrm{Xe}^{19+}$ is observed [Fig. 1(c)] which requires at least 46 EUV photons to be absorbed. Thus, the situation is beyond the scope of low-order perturbation theory and a multiphoton scheme, as discussed previously [2].

Compared to xenon, the high-irradiance spectrum of krypton, the next heaviest rare gas ( $\mathrm{Kr}$ : $[\mathrm{Ar}] 3 d^{10} 4 s^{2}$ $4 p^{6}$ ), looks significantly different as shown in Fig. 2(a). 

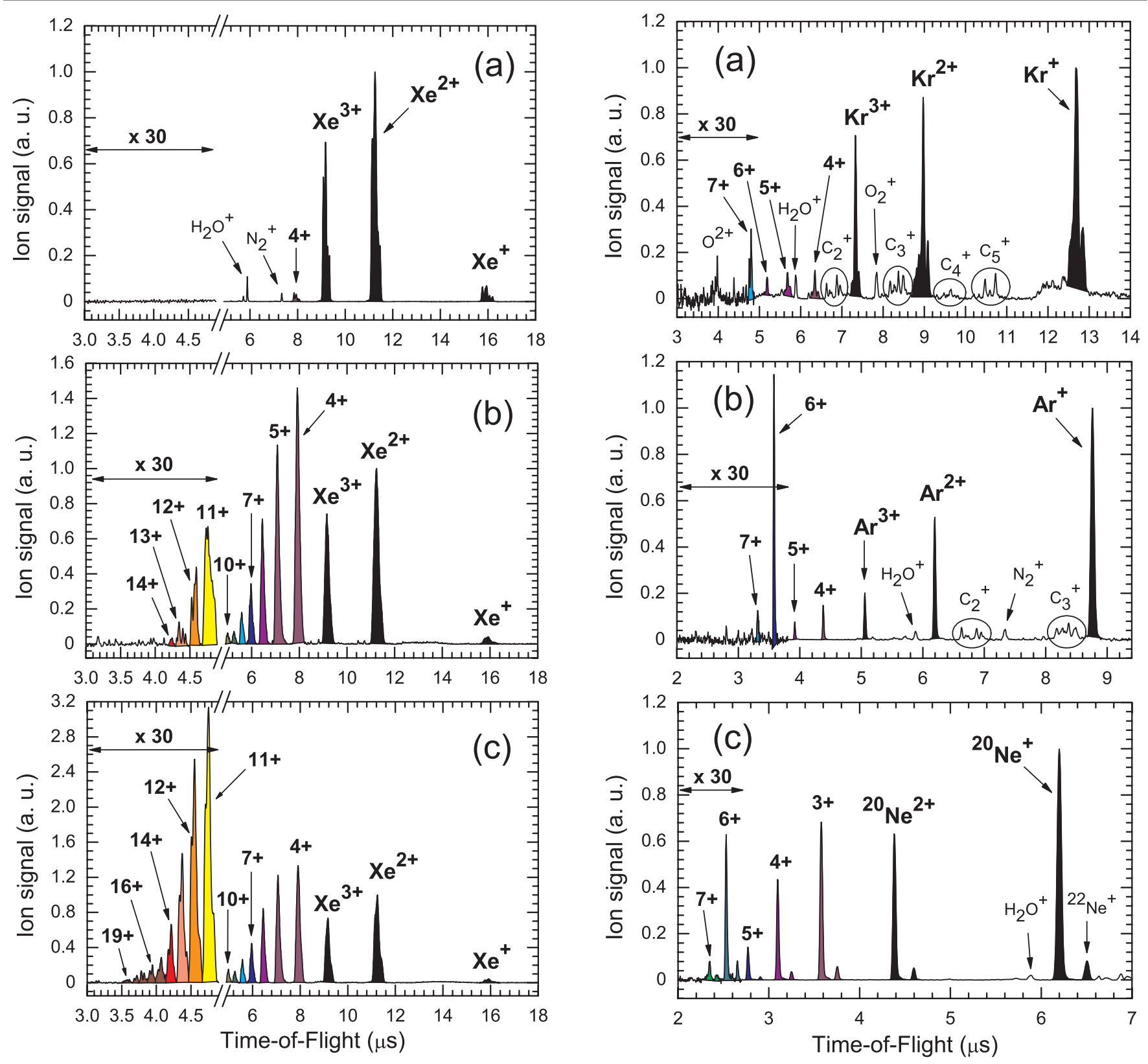

FIG. 1 (color online). Ion time-of-flight (TOF) mass-to-charge spectra of xenon $(\mathrm{Xe})$ taken at $90.5 \mathrm{eV}$ photon energy and irradiance levels of (a) $2.5 \times 10^{12} \mathrm{~W} \mathrm{~cm}^{-2}$, (b) $1.7 \times 10^{15} \mathrm{~W} \mathrm{~cm}^{-2}$, and (c) $2.0 \times 10^{15} \mathrm{~W} \mathrm{~cm}^{-2}$. Signals from residual gas are also indicated.

The highest charge state of $\mathrm{Kr}$ at $(1.5 \pm 0.5) \times$ $10^{15} \mathrm{~W} \mathrm{~cm}^{-2}$ is only $\mathrm{Kr}^{7+}$, which can be simply explained by a sequence of one- and two-photon ionization processes in which an ion created in a preceding step represents the target for a subsequent step. Sequential multiphoton ionization has already been found to be generally dominant at moderate irradiance levels below $10^{14} \mathrm{~W} \mathrm{~cm}^{-2}$ [14,15,21]. The first four rows of Table I summarize, for all rare gases investigated, (a) the highest charge state observable in the spectra of Figs. 1(b) and 2 obtained at irradiance levels in

FIG. 2 (color online). Ion time-of-flight (TOF) mass-to-charge spectra of (a) krypton (Kr), (b) argon (Ar), and (c) neon (Ne), taken at $90.5 \mathrm{eV}$ photon energy and irradiance levels between 1.5 and $1.8 \times 10^{15} \mathrm{~W} \mathrm{~cm}^{-2}$. The $C_{n}{ }^{+}$signals ( $n=2$ to 5 ) possibly arise from carbon clusters desorbed from the carbon coated BL2 focusing mirror.

the range from 1.5 to $1.8 \times 10^{15} \mathrm{~W} \mathrm{~cm}^{-2}$, (b) the ionization energy required to reach this state starting from the atomic ground state [20,22], and (c) the corresponding minimum number $n_{\min }$ of EUV photons of $90.5 \mathrm{eV}$ photon energy which must have been absorbed within a single FEL pulse by an individual atom to deliver this amount of energy. As a result, $n_{\min }$ strongly varies from $n_{\min }=5$ for krypton and argon (Ar: [Ne] $\left.3 s^{2} 3 p^{6}\right), n_{\min }=8$ for neon (Ne: $\left.1 s^{2} 2 s^{2} 2 p^{6}\right)$, and $n_{\min }=22$ for xenon. 
TABLE I. Highest charge state $q^{+}$max observed at irradiance levels in the range from 1.5 to $1.8 \times 10^{15} \mathrm{~W} \mathrm{~cm}^{-2}$, ionization energy $I$ required to reach this state starting from the atomic ground state $[20,22]$ corresponding minimum number $n_{\min }$ of EUV photons of $90.5 \mathrm{eV}$ photon energy which must have been absorbed within a single FEL pulse by an individual atom to deliver this amount of energy, and one-photon ionization cross section $\sigma$ at $90.5 \mathrm{eV}$ photon energy [17] for the rare gases $\mathrm{Ne}$, $\mathrm{Ar}, \mathrm{Kr}$, and $\mathrm{Xe}$, respectively.

\begin{tabular}{lcrcc}
\hline \hline Gas & $q^{+}{ }_{\max }$ & $I / \mathrm{eV}$ & $n_{\text {min }}$ & $\sigma / 10^{-18} \mathrm{~cm}^{2}$ \\
\hline $\mathrm{Ne}$ & $7+$ & 715 & 8 & 4.4 \\
$\mathrm{Ar}$ & $7+$ & 434 & 5 & 1.35 \\
$\mathrm{Kr}$ & $7+$ & 383 & 5 & 0.55 \\
$\mathrm{Xe}$ & $14+$ & 1930 & 22 & 24 \\
\hline \hline
\end{tabular}

With the number of photons absorbed per atom and pulse, the perturbation of the atom by the electromagnetic radiation generally increases. More and more highperturbation orders have to be taken into account to describe the excitation process which may be interpreted as a gradual increase of the wave character of the EUV light on the interaction. It obviously depends strongly on the individual target and its electron structure as demonstrated by our results in Figs. 1 and 2, and Table I. In the optical regime, on the other hand, the wave character of light and the application of nonperturbative theories on the excitation process like the tunneling model and the Keldysh theory are defined by high ponderomotive energy [3-5]:

$$
U_{p} / \mathrm{eV} \approx 1.44 \times \frac{E /\left(10^{13} \mathrm{~W} \mathrm{~cm}^{-2}\right)}{(\hbar \omega / \mathrm{eV})^{2}} .
$$

$U_{p}$ does not depend on the target gas and its individual electron structure but on the characteristics of the incident radiation field only, the irradiance $E$ and photon energy $\hbar \omega$, because it represents the quiver energy transferred from the oscillating field of an electromagnetic wave to the almost free electron of a highly excited Rydberg state. The latter must have been populated by preceding excitation steps. In the EUV, however, photoexcitation into the continuum is the dominant process. Excitation into Rydberg states plays generally a minor role. Thus, nonperturbative theories which are based on ponderomotive motion of highly excited Rydberg electrons seem not to be adequate to describe strong-field phenomena in the EUV which is confirmed by the target dependence of our results. As a consequence, Eq. (1) is not applicable either to define in the EUV the nature of light on the excitation process.

Our comparative study of rare-gas photoionization in the EUV at ultrahigh irradiance levels clearly demonstrates the particular behavior of Xe compared to $\mathrm{Ne}, \mathrm{Ar}$, and $\mathrm{Kr}$. The highest charge state observable by our experiments at almost $2 \times 10^{15} \mathrm{~W} \mathrm{~cm}^{-2}$, the minimum number of photons which must have been almost simultaneously absorbed by an individual atom to reach this state, and, hence, the degree of atomic perturbation by the electromagnetic field are significantly higher for Xe than for the other gases. This is obviously reflected by the respective onephoton ionization cross section values for $90.5 \mathrm{eV}$ photons which are listed in the last row of Table I, known from low photon intensity experiments [17]. In the case of Xe, the photoionization cross section is strongly enhanced by the giant $4 d \rightarrow \varepsilon f$ continuum resonance and amounts to $24 \times 10^{-18} \mathrm{~cm}^{2}$.

In this context, it should be noted that the $4 d$ giant resonance in $\mathrm{Xe}$ and the subsequent elements in the periodic table represent prime examples for strong electron correlation within an atomic system $[23,24]$. The correlations may be described by a collective motion of a full ensemble of quantum particles, where the ten electrons of the $4 d$ shell, driven by the oscillation field of the electromagnetic wave, emit one of their members. The mechanism is in analogy to nuclear giant resonances of protons and neutrons or plasmon excitation in solids and has been applied, for many years, to describe atomic giant resonances excited at low irradiances [23,24]. The application of this idea to high irradiance, on the other hand, can explain our Xe results: due to the higher amplitudes, the collective oscillations within the $4 d$ shell may lead to the emission of more than one $4 d$ electron, up to all ten, more or less simultaneously and coherently. Subsequent Auger decay cascades, then, result in the higher charge states. Such a photoionization scheme represents an extension of the classical photoelectric effect or strong-field ionization in the optical regime which are described by a single photon or an electromagnetic wave interacting just with a single bound outer electron. The scheme might also explain the unexpected irradiance dependence of the higher charge states as discussed in our previous work [2].

The direct multiple ionization of Xe in the inner $4 d$ shell represents, however, a higher order effect and is expected to principally occur at the higher irradiance levels. Moreover, each additional $4 d$ electron simultaneously emitted with its associates should affect the corresponding ion spectrum because it is related with a considerable amount of additional energy transferred to the atom. The sudden rise of higher charge states from $\mathrm{Xe}^{15+}$ to $\mathrm{Xe}^{19+}$ when slightly increasing the irradiance just from 1.7 to $2.0 \times 10^{15} \mathrm{~W} \mathrm{~cm}^{-2}$ as demonstrated by Figs. 1(b) and 1(c) might indicate such step in the direct multiple $4 d$ ionization.

In conclusion, our comparative rare-gas study at FLASH and the particular behavior of xenon show that in the extreme ultraviolet the interaction of high-power lasers with matter cannot be described in the same manner as in the optical regime. Nonperturbative theories based on ponderomotive motion of quasifree electrons are not applicable. On the other hand, the inner-shell electron structure, electron correlation, and resonances play a significant role in explaining strong-field phenomena on photoionization 
in the short-wavelength regime of Einstein's photoelectric effect. First attempts to theoretically approach our xenon results have recently been published $[25,26]$. However, we hope to stimulate further theoretical investigations, in particular, into the role of giant resonances and collective effects on photoionization in the high-intensity shortwavelength regime.

We thank the FLASH team for the very successful operation of the FEL; we also thank J. Costello, P. Lambropoulos, B. Sonntag, G. Ulm, and P. Zimmermann for continuous support and many helpful discussions; support by the Deutsche Forschungsgemeinschaft (DFG) is gratefully appreciated.

*mathias.richter@ptb.de

[1] W. Ackermann et al., Nat. Photon. 1, 336 (2007).

[2] A. A. Sorokin, S. V. Bobashev, T. Feigl, K. Tiedtke, H. Wabnitz, and M. Richter, Phys. Rev. Lett. 99, 213002 (2007).

[3] L. V. Keldysh, Sov. Phys. JETP 20, 1307 (1965).

[4] M. Protopapas, C. H. Keitel, and P. L. Knight, Rep. Prog. Phys. 60, 389 (1997), and references therein.

[5] N. Delone and V. Krainov, Multiphoton Processesin Atoms (Springer, New York, 2000), and references therein.

[6] A. Einstein, Ann. Phys. (Leipzig) 322, 132 (1905).

[7] P. G. O'Shea and H. P. Freund, Science 292, 1853 (2001).

[8] H. C. Kapteyn and T. Ditmire, Nature (London) 420, 467 (2002).
[9] T. Sekikawa, A. Kosuge, T. Kanai, and S. Watanabe, Nature (London) 432, 605 (2004).

[10] J. Feldhaus, J. Arthur, and J. B. Hastings, J. Phys. B 38, S799 (2005).

[11] C. Bostedt et al., Nucl. Instrum. Methods Phys. Res., Sect. A (Kai Siegbahn Memorial Volume) 601, 108 (2009).

[12] T. Feigl, S. Yulin, N. Benoit, and N. Kaiser, Microelectron. Eng. 83, 703 (2006).

[13] A. A. Sorokin et al., Appl. Phys. Lett. 89, 221114 (2006).

[14] A. Sorokin, S. V. Bobashev, K. Tiedtke, and M. Richter, J. Phys. B 39, L299 (2006).

[15] A. Sorokin, M. Wellhöfer, S. V. Bobashev, K. Tiedtke, and M. Richter, Phys. Rev. A 75, 051402(R) (2007).

[16] M. Richter et al., Appl. Phys. Lett. 83, 2970 (2003).

[17] K. Tiedtke et al., J. Appl. Phys. 103, 094511 (2008).

[18] V. Schmidt, Rep. Prog. Phys. 55, 1483 (1992).

[19] T. Luhmann et al., Phys. Rev. A 57, 282 (1998).

[20] K. Yamakawa et al., Phys. Rev. Lett. 92, 123001 (2004).

[21] H. Wabnitz et al., Phys. Rev. Lett. 94, 023001 (2005).

[22] R. L. Kelly, J. Phys. Chem. Ref. Data 16, Suppl. 1, 1 (1987).

[23] Giant Resonances in Atoms, Molecules, and Solids, edited by J.P. Connerade, J. M. Esteva, and R.C. Karnatak (Plenum Press, New York, 1987), and references therein.

[24] M. Ya. Amusia and J.-P. Connerade, Rep. Prog. Phys. 63, 41 (2000).

[25] S. V. Popruzhenko, V. D. Mur, V. S. Popov, and D. Bauer, Phys. Rev. Lett. 101, 193003 (2008).

[26] M. G. Makris, P. Lambropoulos, and A. Mihelic, Phys. Rev. Lett. 102, 033002 (2009). 